TITLE:

\title{
A regularized Boundary Integral Equation Method for Elastodynamic Crack Problems
}

AUTHOR(S):

Nishimura, N.; Kobayashi, S.

\section{CITATION:}

Nishimura, N....[et al]. A regularized Boundary Integral Equation Method for

Elastodynamic Crack Problems. 数理解析研究所講究録 1989, 691: 67-86

ISSUE DATE:

1989-05

URL:

http://hdl.handle.net/2433/101302

RIGHT: 


\title{
A Regularized Boundary Integral Equation Method for Elastodynamic Crack Problems
}
N. Nishimura
and
S. Kobayashi

\section{Department of Civil Engineering, Kyoto University}

\begin{abstract}
This paper presents a double layer potential approach of elastodynamic BIE crack analysis. Our method regularizes the conventional strongly singular expressions for the traction of double layer potential into forms including integrable kernels and 0 th, 1 st and 2nd order derivatives of the double layer density. The manipulation is systematized by the use of the stress function representation of the differentiated double layer kernel functions. This regularization, together with the use of $B$ spline functions, is shown to provide accurate numerical methods of crack analysis in 3D time harmonic elastodynamics.
\end{abstract}

\section{Introduction}

From its early stage of development, the boundary integral equation method has been expected to provide an efficient numerical method of analysis for crack problems. In particular the formulation using double layer potentials appears to be promising because it can deal with cracks of arbitrary shapes, and because it keeps the size of numerical analysis relatively small. Nevertheless, this method has not been investigated very much until recently, because the integration of 
hypersingular kernels in derivatives of double layer potentials was considered to be extremely difficult. Recent efforts to make this computation easier, however, have succeeded either in evaluating these integrals directly (Takakuda et al.(1985), Budreck \& Achenbach(1988)) or in reducing these singular kernels to v.p. (Cauchy's principal value) singular functions (e.g. Bui(1977), Weaver(1977), Sladek \& Sladek(1984), Polch et al.(1987)), or to integrable functions (e.g., the Galerkin formulation by Nedelec(1986) and the collocation method by Nishimura \& Kobayashi(1988)).

In this paper we shall continue the investigation by Nishimura \& Kobayashi(1988) by proposing a new expression for the traction of the double layer potentials in 3D time harmonic elastodynamics with the help of the stress function representation of the differentiated fundamental solution. The new formula for the traction is given in terms of integrable kernel functions and 0 th, 1 st and 2nd order derivatives of double layer densities. In this respect the new formula is similar to the earlier results in Nishimura \& Kobayashi(1988), but is more general in that its extension to other applications such as anisotropy, time domain BIEM, etc. is straightforward. The new regularization formula, together with the use of collocation and $B$ spline, provides 3D BIEM implementations, which take the effect of near tip singularities into consideration by using a change of coordinate. We test our numerical method by solving simple problems and then by comparing the results with known solutions. This paper concludes with comments on the extension of the present formulation to anisotropic cases, and on the relation between Nedelec's variational formulation and the present method. 


\section{Formulation}

Let $S$ be a piece of smooth curved surface in $R^{3}$ bounded by a piecewise smooth edge $\partial S$. Also let $n$ be the unit normal vector to $S$, which points into a side of $S$ called the 'positive side'. Our problem is to find a solution $u$ which satisfies the equations

$$
\begin{aligned}
& \triangle^{*} u+\rho \omega^{2} u=0 \quad \text { in } R^{3} \backslash S, \quad\left(\triangle^{*} u:=\operatorname{div} C[\nabla u]\right) \\
& \mathrm{T} u^{ \pm}=0 \quad \text { on } S, \quad(\mathrm{~T} u:=C[\nabla u] n) \\
& \lim _{x \rightarrow x_{0}}\left(u^{+}(x)-u^{-}(x)\right)=0, \quad x \in S, \quad x_{0} \in \partial S,
\end{aligned}
$$

and radiation condition (Kupradze et al.(1979)) for $u-u_{l}$, where $\Delta^{*}$, T, C, $\rho, \omega$, and $u_{l}$ stand for the Navier operator, elastic traction operator, elasticity tensor, density, frequency, and the incident field which satisfies (1a) in $R^{3}$, respectively. Also, we have used $\mathbf{C}[\nabla \boldsymbol{u}]$ for a tensor whose cartesian components are given by $C_{i j k l}\left(\partial / \partial x_{k}\right) u_{l}$, and a superposed $+(-)$ for a limit from the positive (negative) side of $S$, respectively. The solution $u$ to this problem is complex valued. A physically significant solution is obtained by taking the real part of $u e^{-i \omega t}$.

As is known, the solution $u$ has a double layer representation given by

$$
u(x)=\int_{S} \Gamma_{1}(x, y) \varphi(y) d S+u_{l}(x), \quad x \notin S
$$


where $\Gamma_{l}$ is the double layer kernel defined in terms of the fundamental solution $\Gamma$ as

$$
\Gamma_{I_{i j}}:=\frac{\partial}{\partial y_{q}} \Gamma_{i p}(x-y) C_{j k p q} n_{k}(y)
$$

The fundamental solution of (1a) for the isotropic case is written as (Kupradze et al. (1979))

$$
\Gamma(x-y)=1 F(R)+\nabla \otimes \nabla G(R), \quad(R:=|x-y|)
$$

where

$$
\begin{array}{ll}
F(R)=\frac{e^{i k_{r} R}}{4 \pi \mu R}, & G(R)=\frac{1}{4 \pi \mu k^{2}}\left(\frac{e^{i k_{r} R}}{R}-\frac{e^{i k_{\mathrm{L}} R}}{R}\right), \\
k_{\mathrm{T}}=\frac{\omega}{(\mu / \rho)^{1 / 2}}, & k_{\mathrm{L}}=\frac{\omega}{((\lambda+2 \mu) / \rho)^{1 / 2}},
\end{array}
$$

1 is the unit tensor, and $\lambda$ and $\mu$ are Lame's constants, respectively. Also, C takes the form given by

$$
C_{i j k l}=\lambda \delta_{i j} \delta_{k l}+\mu\left(\delta_{i k} \delta_{j l}+\delta_{i l} \delta_{j k}\right)
$$

For a smooth $\varphi,(1 \mathrm{~b})$ and (2) yield an 'integral' equation

$$
-\mathrm{Tu}(x)=\text { p.f. } \int_{S} \mathrm{~T}_{1}(x, y) \varphi(y) d S, \quad x \in S
$$

where p.f. designates the finite part of a divergent integral.

As one sees easily, the integrand in (7) is hypersingular, making (7) not suitable as a tool of numerical analysis. However, we can reduce the order of the singularity of $T \Gamma_{1}$ by using the integration by part as we shall see. 
To begin with, we consider the static case $(\omega=0)$. We readily see that there exists a stress function $\Phi_{\text {pqrs }}$ which satisfies

$$
\begin{aligned}
& C_{a b i k} \frac{\partial}{\partial x_{k}} \frac{\partial}{\partial y_{l}} \Gamma_{i j}(x-y) C_{c d j l} \\
& =e_{a i p} e_{b j q} e_{c k r} e_{d l s} \frac{\partial}{\partial x_{i}} \frac{\partial}{\partial x_{j}} \frac{\partial}{\partial y_{k}} \frac{\partial}{\partial y_{l}} \Phi_{p q r s}(x-y)+D_{a b c d} \delta(x-y),
\end{aligned}
$$

where $\Gamma_{i j}$ is the fundamental solution of (1a), $e_{i j k}$ is the permutation symbol and $D_{a b c d}$ is a constant, respectively. Indeed, one may view

$$
C_{a b i k} \frac{\partial}{\partial x_{k}} \Gamma_{i j}
$$

as the $(a, b)$ component of the stress associated with $\Gamma_{i j}$, which must be in equilibrium at $x \neq y$. This means that there exists a stress function for the expression in (9). In addition the symmetry of the LHS of (8) with respect to $(a, b)$ and $(c, d)$ requires the existence of the stress function of the form on the RHS of (8) for $x \neq y$. Finally, from the theory of distribution, one shows that the last term in (8) is required for the equality to be valid everywhere in $R^{3}$.

We then use (8) to compute the traction of the double layer potential as follows:

$$
\begin{aligned}
& \int_{S} n_{b}(x) C_{a b i k} \frac{\partial}{\partial x_{k}} \frac{\partial}{\partial y_{l}} \Gamma_{i j}(x-y) C_{c d j l} n_{c}(y) \varphi_{d}(y) d S \\
& =-\int_{S} n_{b}(x) e_{a i p} e_{b j q} e_{d l s} \frac{\partial}{\partial x_{i}} \frac{\partial}{\partial x_{j}} \frac{\partial}{\partial y_{l}} \Phi_{p q r s}\left(n_{c} e_{c k r} \varphi_{d, k}\right) d S \\
& =-\int_{S}\left(n_{b}(x)-n_{b}(y)\right) e_{a i p} e_{b j q} e_{d l s} \frac{\partial}{\partial x_{i}} \frac{\partial}{\partial x_{j}} \frac{\partial}{\partial y_{l}} \Phi_{p q r s}\left(n_{c} e_{c k r} \varphi_{d, k}\right) d S
\end{aligned}
$$




$$
\text { - p.f. } \int_{S} e_{a i p} e_{d l s} \frac{\partial}{\partial x_{i}} \frac{\partial}{\partial y_{l}} \Phi_{p q r s} n_{b} e_{b j q} \frac{\partial}{\partial y_{j}}\left(n_{c} e_{c k r} \varphi_{d . k}\right) d S
$$

where $x \notin S$, and the normal vector $n(x)$ is arbitrary as far as it approaches the positive normal vector to $S$ as $x$ tends to a point on $S$. The p.f. symbol on the RHS of (10) is due to the singularity of $\nabla \nabla \varphi$ on $\partial S$. It is seen that the kernel functions on the RHS of have integrable singularities at $x=y$ if $S$ is smooth. Indeed, the and (3rd) derivatives of $\Phi$ are $O\left(|x-y|^{-1}\right)\left(O\left(|x-y|^{-2}\right)\right), \quad$ and $n(x)-n(y)=O(|x-y|)$ as $|x-y| 10$. Therefore the limit of (10) as $x \rightarrow S$ takes exactly the same form as (10) because all the kernels in (10) are integrable. We thus see that the regularization of (7) is straightforward once one gets the stress function $\Phi$ in (8).

We now proceed to compute $\Phi$. This calculation is made easier with the help of the Fourier transform. In 3D isotropic case the Fourier transform of

$$
-C_{a b i k} \frac{\partial}{\partial x_{k}} \frac{\partial}{\partial x_{l}} \Gamma_{i j}(x) C_{c d j l}
$$

is written as

$$
\begin{aligned}
& \frac{1}{(\lambda+2 \mu)|\xi|^{4}}\left\{\lambda^{2}|\xi|^{4} \delta_{a b} \delta_{c d}+2 \lambda \mu|\xi|^{2}\left(\xi_{a} \xi_{b} \delta_{c d}+\xi_{c} \xi_{d} \delta_{a b}\right)\right. \\
& +\mu(\lambda+2 \mu)|\xi|^{2}\left(\delta_{a c} \xi_{b} \xi_{d}+\delta_{b d} \xi_{a} \xi_{c}+\delta_{a d} \xi_{b} \xi_{c}+\delta_{b c} \xi_{a} \xi_{d}\right) \\
& \left.-4 \mu(\lambda+\mu) \xi_{a} \xi_{b} \xi_{c} \xi_{d}\right\}
\end{aligned}
$$

where $\xi$ is the parameter of the Fourier transform. But (12) is equal to 


\section{$\frac{1}{(\lambda+2 \mu)|\xi|^{4}}\left\{\left(2 \lambda \mu e_{a i m} e_{b j m} e_{c k n} e_{d l n}\right.\right.$}

$$
\left.+\mu(\lambda+2 \mu)\left(e_{a i m} e_{c j m} e_{b k n} e_{d l n}+e_{a i m} e_{d j m} e_{b k n} e_{c l n}\right)\right\} \xi_{i} \xi_{j} \xi_{k} \xi_{l}
$$

modulo a constant tensor, whose Fourier inverse transform is proportional to Dirac's delta. Hence we use (8) and (13) to have

$$
\begin{gathered}
\Phi_{s t u v}=-\frac{\mu}{8 \pi(\lambda+2 \mu)}\left\{2 \lambda \delta_{s t} \delta_{u v}+(\lambda+2 \mu)\left(\delta_{s u} \delta_{t v}+\delta_{s v} \delta_{t u}\right)\right\} \\
\cdot|x-y| .
\end{gathered}
$$

We now consider the dynamic case. Since the elastodynamic counterpart of (12) differs from (12) by a function of order $1 /|\xi|^{2}$ as $|\xi| \rightarrow \infty$, we generally have

$$
\begin{aligned}
& C_{a b i k} \frac{\partial}{\partial x_{k}} \frac{\partial}{\partial y_{i}} \Gamma_{i j}(x-y) C_{c d j l} \\
& =e_{a i p} e_{b j q} e_{c k r} e_{d l s} \frac{\partial}{\partial x_{i}} \frac{\partial}{\partial x_{j}} \frac{\partial}{\partial y_{k}} \frac{\partial}{\partial y_{l}} \Phi_{p q r s}^{*}(x-y)+\Psi_{a b c d}^{*}(x-y) \\
& +D_{a b c d}^{*} \delta(x-y)
\end{aligned}
$$

where $\Phi^{*}$ is a function of order $|x-y|$ as $|x-y| 10, \Psi^{*}$ is a function which has an integrable singularity at $x=y$ and $D_{a b c d}^{*}$ is a constant tensor. Of course, $\Gamma_{i j}$ in (15) indicates the elastodynamic fundamental solution. The functions $\Phi^{*}$ and $\Psi^{*}$ in (15) are not determined uniquely. For example one may use the elastostatic $\Phi$ for $\Phi^{*}$, or alternatively use the following in the isotropic case: 


$$
\begin{aligned}
& C_{a b i k} \frac{\partial}{\partial x_{k}} \frac{\partial}{\partial y l} \Gamma_{i j}(x-y) C_{c d j l} \\
& \sim \frac{1}{4 \pi(\lambda+\mu)}\left\{\mu k^{2}\left\{\lambda \delta_{a b} \delta_{c d}+\mu\left(\delta_{a c} \delta_{b d}+\delta_{a d} \delta_{b c}\right)\right\}\left(\frac{k^{2} T}{k^{2}} \frac{e^{i k_{r} R}}{R}-\frac{k^{2}}{k_{T}^{2}} \frac{e^{i k_{\iota} R}}{R}\right)\right. \\
& -\mid \lambda^{2} \delta_{a b} \delta_{c d} \delta_{s t}+2 \lambda \mu\left(\delta_{a b} \delta_{c s} \delta_{d t}+\delta_{c d} \delta_{a s} \delta_{b t}\right) \\
& \left.+\mu^{2}\left(\delta_{a c} \delta_{b s} \delta_{d t}+\delta_{a d} \delta_{b s} \delta_{c t}+\delta_{b d} \delta_{a s} \delta_{c t}+\delta_{b c} \delta_{a s} \delta_{d t}\right)\right\} \\
& \frac{\partial}{\partial x_{s}} \frac{\partial}{\partial y_{t}}\left(\frac{e^{i k_{r} R}}{R}-\frac{e^{i k_{\mathrm{L}} R}}{R}\right) \\
& -\frac{\mu}{k_{T}^{2}}\left\{2 \lambda \delta_{p q} \delta_{r s}+(\lambda+2 \mu)\left(\delta_{p r} \delta_{q s}+\delta_{p s} \delta_{q r}\right)\right\} \\
& \left.e_{a i p} e_{b j q} e_{c k r} e_{d l s} \frac{\partial}{\partial x_{i}} \frac{\partial}{\partial x_{j}} \frac{\partial}{\partial y_{k}} \frac{\partial}{\partial y_{l}}\left(\frac{e^{i k_{r} R}}{R}-\frac{e^{i k_{\iota} R}}{R}\right)\right\}
\end{aligned}
$$

where $R=|x-y|$, and $\sim$ indicates an equality modulo Dirac's delta. The first 4 lines on the RHS of (16) are identified as $\Psi_{a b c d}^{*}$. It is now a simple matter to regularize the traction of the elastodynamic double layer potential. Indeed, we have

$$
\begin{aligned}
& \int_{S} n_{b}(x) C_{a b i k} \frac{\partial}{\partial x_{k}} \frac{\partial}{\partial y_{l}} \Gamma_{i j}(x-y) C_{c d j l} n_{c}(y) \varphi_{d}(y) d S \\
& =-\int_{S}\left(n_{b}(x)-n_{b}(y)\right) e_{a i p} e_{b j q} e_{d l s} \frac{\partial}{\partial x_{i}} \frac{\partial}{\partial x_{j}} \frac{\partial}{\partial y_{l}} \Phi_{p q r s}^{*}\left(n_{c} e_{c k r} \varphi_{d, k}\right) d S \\
& - \text { p.f. } \int_{S} e_{a i p} e_{d l s} \frac{\partial}{\partial x_{i}} \frac{\partial}{\partial y_{l}} \Phi_{p q r s}^{*} n_{b} e_{b j q} \frac{\partial}{\partial y_{j}}\left(n_{c} e_{c k r} \varphi_{d, k}\right) d S
\end{aligned}
$$




$$
+\int_{S} n_{b}(x) \Psi_{a b c d}^{*} n_{c}(y) \varphi_{d} d S
$$

In passing, we note that (16) leads to a variational statement similar but not identical to the expression used by Nedelec(1986).

Equations (16) and (17) are considered to be useful as the basis of numerical analyses because all the singularities of the integrands in (17), except for the near tip singularities of $\nabla \nabla \varphi$, are integrable. In addition, there is an easy way of evaluating the p.f. integrals in. (17), as we shall see. Finally, we note, in the flat crack case, that the 2nd integral on the RHS of (17) vanishes as $x$ tends to $S$. Also, one shows that the in plane and out of plane components of (17) decouple as one computes the derivatives in (17) explicitly by using (16) .

\section{Numerical analysis}

The numerical method used here is almost identical with those used in Nishimura \& Kobayashi(1988). In this section, however, we shall outline our numerical method for the purpose of completeness. As in Nishimura \& Kobayashi(1988), we shall discretize (7) by using (17) and the collocation method, which is believed to be efficient in engineering applications. With this choice, however, it is preferable to use $C^{\prime}$ base functions. Indeed, piecewise constant base functions for $\varphi$ are likely to pollute the solution by introducing $1 / \delta$ singularities in (17), where $\delta$ is the distance between the observation point $x$ and a point where $\varphi$ is discontinuous. Actually, the second 
derivatives of $\varphi$ in (17) would behave like the 1st derivatives of Dirac's delta, which would pick up singularities from the kernel functions. Still worse is a piecewise linear approximation, in which case (17) would diverge logarithmically at points where the slope of the base functions are discontinuous. This means that a nodal collocation with piecewise linear base functions breaks down. Hence we are left with the use of $C^{\prime}$ base functions with which (17) is seen to remain bounded on $S$.

This restriction of $C^{\prime}$ continuity is not very stringent in a special case where one can find a smooth mapping from $S$ onto a square, say $\left|x_{1}\right|<a,\left|x_{2}\right|<a, x_{3}=0$. Indeed, one first generates B-spline functions of $x_{1}$ and $x_{2}$ independently in the following manner:

1) Introduce a parameter $t$ such that $s=a \sin (\pi t / 2 a)(|t|<a)$ where $s$ is either $x_{1}$ or $x_{2}$.

2) Divide the interval $(-a, a)$ for $t$ into subintervals of equal length $\Delta t$ by using nodes $\left(-a \Rightarrow t_{0}<t_{1}<\ldots<t_{n+1}(=a)\right.$.

3) Define 3rd order spline functions $\sigma_{i}(t) \quad(i=1,2, \ldots, n)$ by $\sigma_{i}(t)=f\left(\left|t-t_{i}\right| / \Delta t\right)(i=1, \ldots, n) \quad$ with exceptions $\sigma_{1}(t)=g\left(\left|t-t_{1}\right| / \Delta t\right)$ for $t_{0}<t<t_{1}$ and $\sigma_{n}(t)=g\left(\left|t-t_{n}\right| / \Delta t\right)$ for $t_{n}<t<t_{n+1}$ where (see Fig. 1)

$$
\begin{aligned}
& f(\eta)= \begin{cases}1-(3 / 2) \eta^{2}+(3 / 4) \eta^{3} & 0 \leqq \eta<1, \\
(1 / 4)(2-\eta)^{3} & 1 \leqq \eta<2, \\
0 & \text { otherwise, }\end{cases} \\
& g(\eta)=(3 / 2)(1-\eta)-(1 / 2)(1-\eta)^{3} \quad 0 \leqq \eta<1 .
\end{aligned}
$$




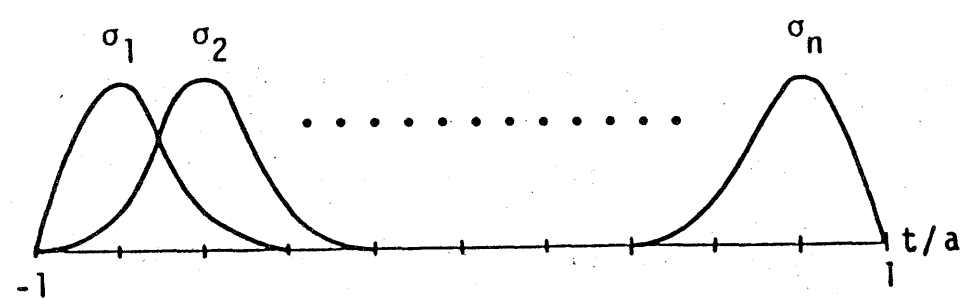

Fig.1 Spline functions

With the spline functions of $x_{1}$ and $x_{2}$ thus defined, we can now proceed to construct a system of base functions on the square by multiplying the $x_{1}$ and $x_{2}$ spline functions together. Namely, we use $\sigma_{i}\left(t\left(x_{1}\right)\right) \sigma_{j}\left(t\left(x_{2}\right)\right)$ as the base functions. Finally one determines the base functions $\Omega_{i}(x)$ on $S$ by using the composition of the mapping from $S$ to the square and the base functions on the square which we have just defined.

Now assume

$$
\varphi=\sum \varphi_{i} \Omega_{i}(x)
$$

where $\varphi_{i}$ are numbers. We substitute (20) into (17) to discretize (7), and solve the resulting numerical matrix for $\varphi_{i}$ to determine $\varphi$ with the help of (20). Note that $\varphi(x)$ for a point $x$ near $\partial S$ is asymptotically proportional to the square root of the distance from the edges of the crack to $x$. This is due to the relation between $s$ and $t$. Hence one easily obtains stress intensity factors by computing the coefficients of square root singular terms in $\varphi$. At corner points, however, this method renders $\varphi$ non singular. The exact solution, on the other hand, is known to be weakly singular there with an exponent which is greater than $1 / 2$ and is dependent on Poisson's ratio (e.g. Takakuda(1985)). This neglecting of the corner singularity, however, 
does not seem to have a significant effect on the overall accuracy of the numerical solution as we shall see through examples.

Another practically important special case which allows a simple implementation is the case where there exists a smooth mapping from $S$ onto a disc. By the same reasoning as before we are justified to restrict our attention to the case of a penny shaped crack having a radius of a. Our scheme for this case goes as follows:

1) Introduce $(t, \theta)$ coordinate system by which the natural polar coordinate on the crack, denoted by $(r, \theta)$, is expressed as $(r, \theta)=(a \sin (\pi t / 2 a), \theta)$. We also define $\left(\xi_{1}, \xi_{2}\right)=(t \cos \theta, t \sin \theta)$.

2) Divide the interval $(0, a)$ for $t$ into subintervals of equal length $\Delta t$ by introducing nodes $\left(0 \Rightarrow t_{0}<t_{1}<\ldots<t_{n+1}(=a)\right.$. Also divide $(0,2 \pi)$ for $\theta$ into subintervals of equal length $\left(0 \Rightarrow \theta_{0}<\theta_{1}<\ldots<\theta_{m}(=2 \pi)\right.$. For nodes $\left(t_{i}, \theta_{j}\right)$ ( $2 \leqq i \leqq n, 1 \leqq j \leqq m)$ we use 'product' base functions similar to those used before. This determines $(n-1) m$ base functions $\Omega_{i}(x)$.

3) In the domain defined by $(t, \theta), t \leqq t_{1}, 0 \leqq \theta<2 \pi$, we take points $\xi^{i}(1 \leqq i \leqq k)$ arbitrarily. Draw circles of arbitrary (but not too large) radius $2 r_{i}$ centred at $\xi^{i}$. Define 'bell shaped' base functions associated with $\xi^{i}$ by $\Omega_{(n-1) m+i}(x)=f\left(\left|\xi-\xi^{i}\right| / r_{i}\right)$. A standard choice for $r_{i}$ is $\Delta t$.

4) Use the base functions defined in 2) and 3) (see Fig.2), assume (20) for $\varphi$, and then discretize (7) with the help of (17). 


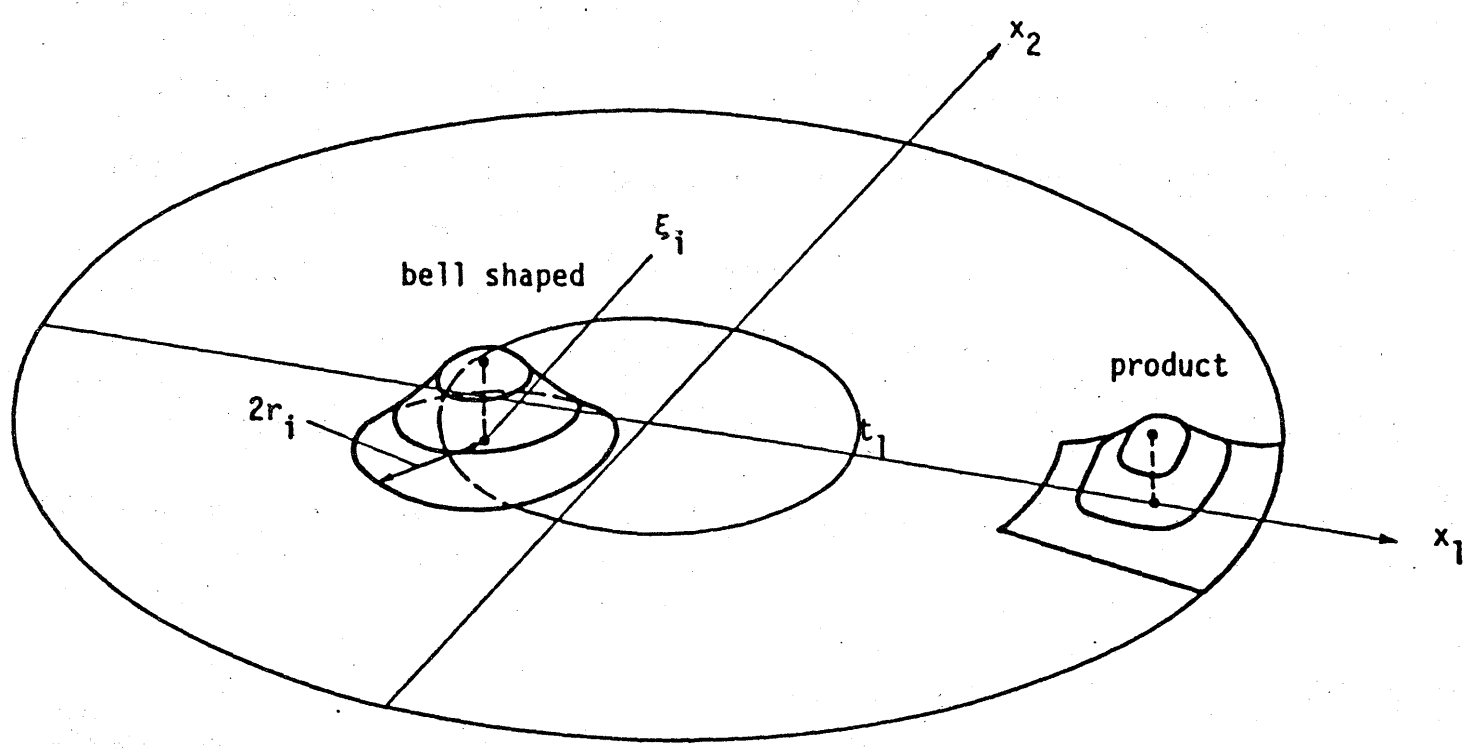

Fig.2 Base functions for penny shaped crack

Finally we make several comments concerning our methods. We first note that $\varphi_{i}$ in (20) does not have a clear physical meaning since $\Omega_{i}\left(x_{j}\right) \neq \delta_{i j}$. This is why we prefer the name 'base functions' for our $\Omega_{i}$. The second comment is related to the method of evaluating the remaining p.f. integral in (17). In order to explain our method we take the second special case as an example. We shall start with a formula

$$
\begin{aligned}
& \text { p.f. } \int_{0}^{2 \pi} \int_{0}^{a}\left(\frac{\partial}{\partial r}\right)^{2} \Omega(r, \theta) H(r, \theta) r d r d \theta \\
& =\int_{0}^{2 \pi} \int_{0}^{a}\left(\frac{\partial}{\partial r}\right)^{2} \Omega(r, \theta)(r H(r, \theta)-a H(a, \theta)) d r d \theta
\end{aligned}
$$


where $\Omega$ stands for one of the base functions having singularities on $\partial S\left((\partial / \partial r)^{2} \Omega \sim(a-r)^{-3 / 2}\right)$, and $H$ is a function regular at $r=a$. In deriving (21) we have used the fact that $\Omega$ vanishes except in a region near the tip. With (21) we can compute the p.f. integrals in (17) numerically since they take the form of the LHS of (21), and the RHS of (21) converges in the ordinary sense. For evaluating an integral having an integrable but weakly singular integrand, we use changes of coordinates used by Lachat \& Watson(1976) and by Watson(1982), together with the Gaussian integration. Finally, we remark that the $\left(e^{i k_{r} R} / R-e^{i k_{\iota} R} / R\right)$ terms in (16) should be evaluated by using series expansions when $k_{T} R$ is small. This is in order to avoid cancellation, as has been discussed in Kobayashi \& Nishimura(1982).

\section{Numerical results}

We consider a square crack having side length of $2 a$ subject to a plane $P$ wave of normal incidence. The stress magnitude of the incident wave is $p_{0}$. This problem has been investigated by Itou(1980). Our analysis has used a mesh having 169 nodes. Poisson's ratio is set equal to 0.2 . Fig. 3 shows the stress intensity factor along an edge of the crack. Fig. 4 plots the crack opening displacement $\varphi_{3}$ on $x_{1}=x_{3}=0$ in the cartesian coordinate shown in the same figure. These figures show that the results of the present analysis (symbols), agree well with the numerical results by Itou (lines). 


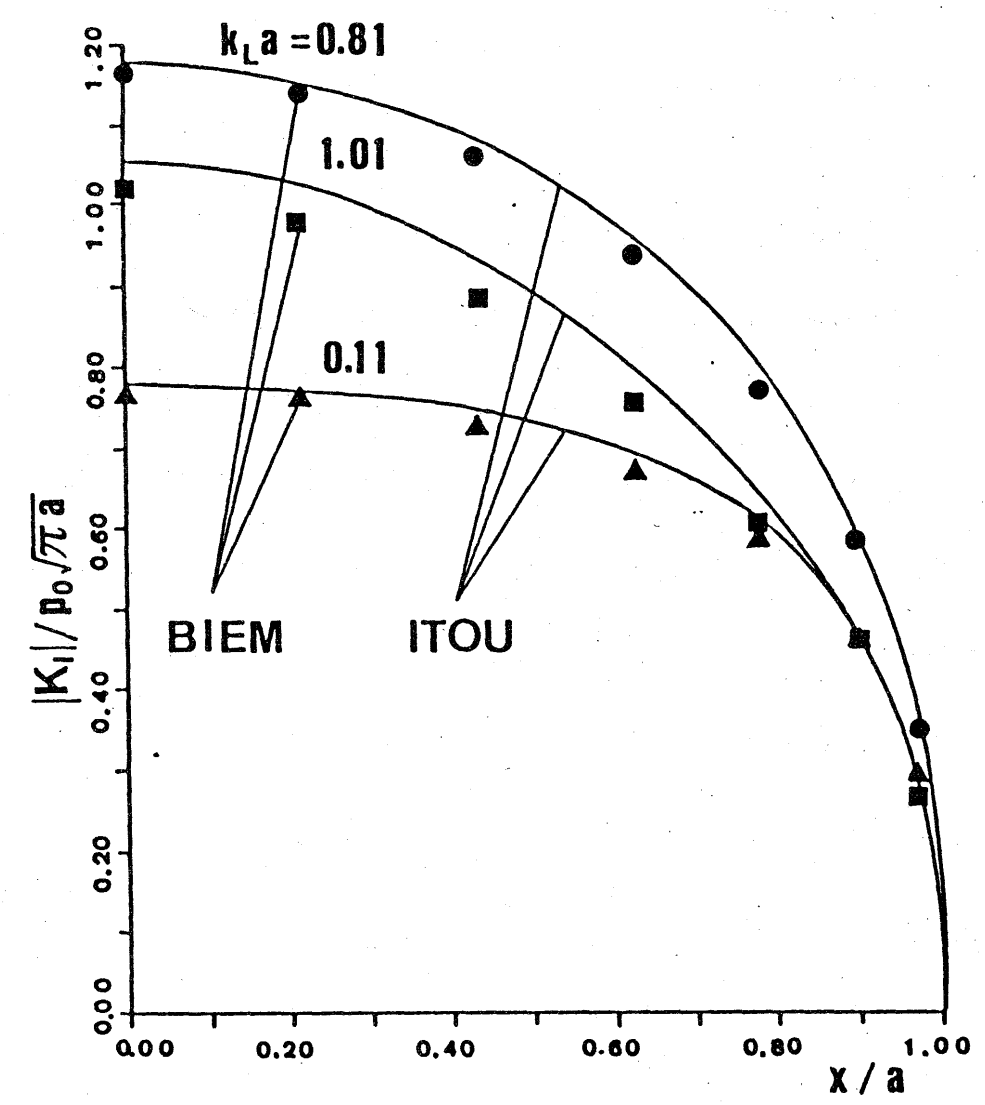

Fig.3 Stress intensity factor along an edge of a square crack

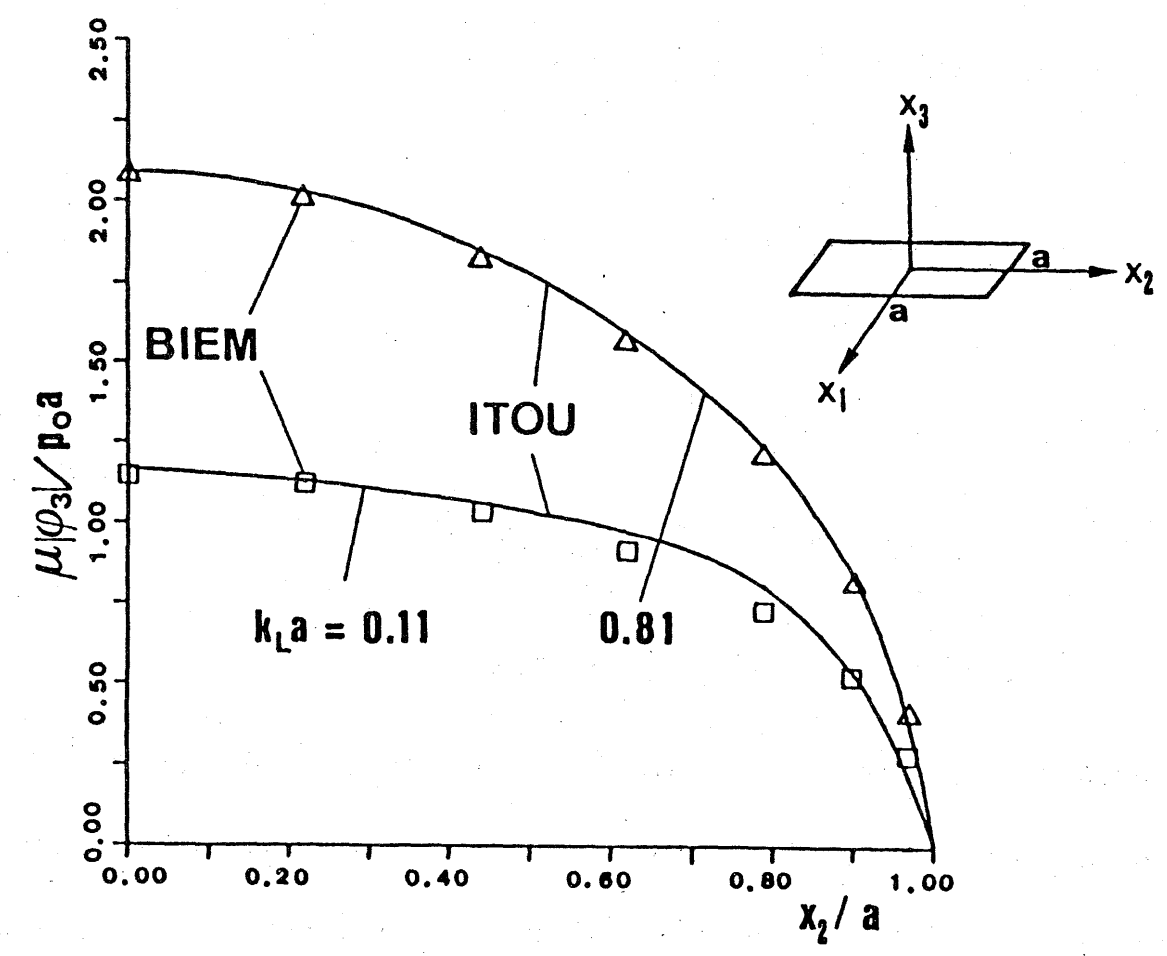

Fig.4 Crack opening displacement of a square crack 


\section{Concluding remarks}

1) In statics, a stress function exists for an arbitrary equilibrated stress field. This means that the regularization is possible regardless of the material symmetry, although it is not very easy to obtain the explicit forms of the stress functions similar to (16) in the general 3D anisotropic case. In $2 \mathrm{D}$ case, however, it is not difficult to obtain the general formulae for the stress functions even in the fully anisotropic case. Indeed, we have

$$
\begin{aligned}
\mathcal{F}( & \left.-C_{a b i k} \frac{\partial}{\partial x_{k}} \frac{\partial}{\partial x_{l}} \Gamma_{i j} C_{j l c d}\right) \\
& =1-\frac{1}{2} e_{a i} e_{b j} e_{c k} e_{d l} \xi_{i} \xi_{j} \xi_{k} \xi_{l} \operatorname{det} \mathrm{C} \\
& \left.+\rho \omega^{2}\left(C_{a b c d} C_{i j i l}-C_{i j a b} C_{i l c d}\right) \xi_{j} \xi_{l}-\rho^{2} \omega^{4} C_{a b c d}\right) / J
\end{aligned}
$$

where $\mathcal{F}$ stands for the Fourier transform and

$$
\begin{aligned}
J_{0}= & \frac{1}{2} C_{i j k l} \xi_{j} \xi_{l} C_{p q r s} \xi_{q} \xi_{s} e_{i p} e_{k r}, \\
J= & J_{0}-\rho \omega^{2} C_{i j i l} \xi_{j} \xi_{l}+\rho^{2} \omega^{4} \\
\operatorname{det} C= & 2\left(C_{11111} C_{2222} C_{1212}+2 C_{1122} C_{1222} C_{1112}\right. \\
& \left.\quad-C_{1112}^{2} C_{2222}-C_{1122}^{2} C_{1212}-C_{1222}^{2} C_{1111}\right)
\end{aligned}
$$

The Fourier inversion of (२2) for the static orthotropic case can be carried out explicitly by using standard techniques (e.g. Green \& Zerna(1954)). The dynamic counterpart, however, is possible only in the time domain. Namely, the Fourier inversion of (२2) in the 
orthotropic case is possible if one takes $\omega$ to be the parameter of the Fourier transform with respect to time. In this paper we shall not go into further detail of the regularization process in time domain because this subject has been discussed elsewhere (Nishimura \& Kobayashi(1987) and Guo et al.(1987)).

2) The present formulation is closely related to the variational formulation by Nedelec(1986). To see the relation between his formulation and ours, we remember that our method moves some derivatives in $T \Gamma_{1}$ to the double layer density by using integration by part. Nedelec's formulation starts by multiplying (7) by an arbitrary weight function $w_{i}$ such that $w_{i}=0$ on $\partial S$, followed by integration of the resulting expression over $S$. Integration by part then transfers some of the derivatives in $T \Gamma_{1}$ to the double layer density and others to the weight function, thus yielding Nedelec's variational formulation. Some of the advantages of Nedelec's formulation are: 1) The resulting matrix equation is symmetric and good conditioned. 2) One may use $C^{0}$ base functions for computing the crack opening displacement. The present method, on the other hand, is less flexible than his as far as the choice of base functions is concerned because our method breaks down with piecewise linear elements, for example. However, our method is presumably faster than his method since ours uses collocation.

3) The motivation for the use of $C^{\prime}$ elements in our method has been to eliminate the non-physical singularities of the traction on the crack face. This choice has made the use of higher order elements possible, but at the same time has set a limit to the possible shape of cracks. 


\section{4}

When an approximation $\varphi^{\prime}{ }_{i}$ for the crack opening displacement $\varphi_{i}$ is available, however, one may introduce a new unknown function $\psi_{i}$ in terms of which the real crack opening displacement $\varphi_{i}$ is expressed as

$$
\left.\varphi_{i}=\varphi^{\circ} \psi_{i}, \quad \text { (no sum over } i\right)
$$

and then use piecewise constant approximation for $\psi_{i}$. For example one may use the exact static solution, if available, for $\varphi^{\circ} i$ in carrying out an elastodynamic analysis for a small $\omega$. This approximation does introduce $1 / \delta$ singularities into the numerical solution, as discussed in 3. However, the effect of these singularities on the accuracy of the solutions at collocation points may not be very important when the solution $\psi_{i}$ is expected to be almost constant over $S$. An attempt along this line in time domain is found in Guo et al.(1987).

\section{Acknowledgement}

The authors wish to express their gratitude to Messrs. K. Matsumoto and N. Nakamura for their numerical work, and Prof. H. Horii of University of Tokyo for his comments on the use of piecewise constant elements.

\section{References}

1. Budreck, D.E. \& Achenbach, J.D.(1988): Scattering from three-dimensional planar cracks by the boundary integral equation method. submitted to J. Appl. Mech.

2. Bui, H.D.(1977): An integral equations method for solving the 
problem of a plane crack of arbitrary shape. J. Mech. Phys. Solids. 25, 29-39

3. Green, A.E. \& Zerna, W.(1954): Theoretical Elasticity. London: Oxford Univ. Press

4. Guo, Q.C., Nishimura, N. \& Kobayashi, S. (1987): Elastodynamic analysis of a crack by BIEM. Proc. 4th Japan Nat. Symp. BEM. 197-202 (in Japanese)

5. Itou, S. (1980): Dynamic stress concentration around a rectangular crack in an infinite elastic medium. ZAMM. 60, 381-388

6. Kobayashi, S. \& Nishimura, N. (1982): Transient stress analysis of tunnels and cavities of arbitrary shape due to travelling waves. In: Banerjee, P.K. \& Shaw, R.P. (Eds): Developments in Boundary Element Methods - II, pp. 177-210. London: Applied Science Publishers

7. Kupradze, V.D., Gegelia, T.G., Basheleishvili, M.0. \& Burchuladze, T.V.(1979): Three-Dimensional Problems of the Mathematical Theory of Elasticity and Thermoelasticity. Amsterdam: North-Holland.

8. Lachat, J.C. \& Watson, J.O.(1976): Effective numerical treatment of boundary integral equations. Int. J. Num. Meth. Eng. 10, $991-1005$

9. Nedelec, J.C.(1986): The double layer potential for periodic elastic waves in $R^{3}$. In: Du, Q. (Ed): Boundary Elements. pp. 439-448. Oxford: Pergamon Press

10. Nishimura, N. \& Kobayashi, S. (1987): On the regularisation of the differentiated double layer potentials in elasticity. Proc. 4th Japan Nat. Symp. BEM. 49-54 (in Japanese)

11. Nishimura, N. \& Kobayashi, S. (1988): An improved boundary integral equation method for crack problems. In: Cruse, T.A.(Ed): Proc. 
IUTAM Symposium on Advanced Boundary Element Methods. to appear

12. Polch, E.Z., Cruse, T.A. \& Huang, C.-J.(1987): Traction BIE solutions for flat cracks, Computational Mech. 2, 253-267

13. Sladek, V. \& Sladek, J.(1984): Transient elastodynamic three-dimensional problems in cracked bodies. Appl. Math. Model. $8,2-10$

14. Takakuda, K. (1985): Stress singularities near crack front edges: Bull. JSME. 28, 225-231

15. Takakuda, K., Koizumi, T. \& Shibuya, T. (1985): On integral equation methods for crack problems. Bull. JSME. २8, 217-224

16. Watson, J.O. (1982): Hermitian boundary elements for plane problems of fracture mechanics. Res Mech. 4, 23-42

17. Weaver, J.(1977): Three dimensional crack analysis. Int. J. Solids Structures. $13,321-330$ 